\title{
Avaliação da acupressão auricular na Síndrome do Ombro Doloroso: estudo de caso
}

\author{
Evaluation of ear acupressure on Painful Shoulder Syndrome: case study \\ Evaluación de la acupresión auricular sobre el Síndrome de Hombro Doloroso: estudio de caso
}

\begin{abstract}
Ana Paula Zanelatto'
' Universidade Cruzeiro do Sul, Curso de Graduação de Enfermagem. São Paulo-SP, Brasil.
\end{abstract}

Submissão: 25-03-2012 Aprovação: 22-07-2013

\section{RESUMO}

A Síndrome do Ombro Doloroso (SOD) é definida como dor e limitação funcional decorrente do acometimento de estruturas estáticas e dinâmicas do ombro, como ligamentos, cápsula e músculos. É uma das queixas mais comuns e incapacitantes do sistema musculoesquelético na população em geral com uma prevalência estimada entre 15 a $25 \%$. Este estudo objetivou avaliar os resultados da acupressão auricular quando usada como terapia no tratamento da Dor crônica, secundária à SOD, quanto ao efeito analgésico e terapêutico satisfatório e o tempo estimado de tratamento. Para a obtenção do objetivo foi realizado um Estudo de Caso que teve como intervenção a acupressão auricular com esferas de cristais e como indicador de resultado o questionário Penn Shoulder Score (PSS - Brasil). Os dados foram analisados qualitativa e quantitativamente. Concluise que a auriculoterapia é uma importante técnica terapêutica, pois o estudo demonstrou uma melhora na pontuação total do PSS - Brasil em 34,3\%.

Descritores: Síndrome do Ombro Doloroso; Auriculoterapia; Acupuntura; Terapias Complementares; Diagnóstico de Enfermagem.

ABSTRACT

The Painful Shoulder Syndrome (PSS) is defined as pain and functional limitation resulting from impairment of static and dynamic structures of the shoulder, such as ligaments, capsule and muscles. It is one of the most common and disabling of the musculoskeletal system in the general population with prevalence estimated between 15-25\%. This study aimed to evaluate the results of auricular acupressure when used as therapy in the treatment of chronic pain secondary to SOD, and the analgesic effect and therapeutic satisfactory and the estimated time of treatment. To obtain the objective was to perform a case study was to intervene with auricular acupressure beads and crystals as an indicator of the outcome questionnaire Penn Shoulder Score (PSS - Brazil). Data were analyzed qualitatively and quantitatively. We conclude that auriculotherapy is an important therapeutic technique, because the study showed an improvement in the total score of the PSS - Brazil at $34.3 \%$.

Key words: Painful Shoulder Syndrome; Auriculotherapy; Acupuncture; Complementary Therapies; Nursing Diagnosis.

\section{RESUMEN}

El síndrome de hombro doloroso (SHD) se define como dolor y limitación funcional que resulta del deterioro de las estructuras estáticas y dinámicas de los hombros, como ligamentos, cápsula y los músculos. Es uno de los más comunes y incapacitantes del sistema músculo-esquelético en la población general con una prevalencia estimada entre el $15-25 \%$. Este estudio tuvo como objetivo evaluar los resultados de la digito presión auricular cuando se usa como terapia en el tratamiento del dolor crónico secundario a la SHD, y el efecto analgésico y terapéutico satisfactorio y el tiempo estimado de tratamiento. Para lograr el objetivo ha sido realizar un estudio de caso Estudo de Caso en que se tuve como intervención la acupressão auricular con esferas de cristales y como indicador de resultado el cuestionario Penn Shoulder Score (PSS - Brasil). Los datos se analizaron cualitativa y cuantitativamente. Llegó-se a la conclusión de que la auriculoterapia es una técnica terapéutica importante, porque el estudio demostró una mejoría en la puntuación total de la PSS - Brasil en el 34,3\%.

Palabras clave: Síndrome del Hombro Doloroso; Auriculoterapia; Acupuntura; Las Terapias Complementarias; Diagnóstico de Enfermería. 


\section{INTRODUÇÃO}

A Síndrome do Ombro Doloroso (SOD) é definida por dor e limitação funcional decorrente do acometimento de estruturas estáticas e dinâmicas do ombro, como ligamentos, cápsula e músculos. É uma das queixas mais comuns e incapacitantes do sistema musculoesquelético na população em geral. Tem uma prevalência estimada entre 15 a $25 \%$ e, atualmente, é a segunda causa de queixa de dor no aparelho locomotor, perdendo apenas para a dor na coluna vertebral. Pode acometer pessoas de qualquer faixa etária, porém sua incidência aumenta com o processo de envelhecimento, em indivíduos esportistas ou aqueles que utilizam os membros superiores em ocupações laborativas ${ }^{(1-3)}$.

De maneira geral, SOD é tratada de forma conservadora tais como cirurgias (em casos específicos), medicamentos e fisioterapia. Quando não tratada, leva a longos períodos de dor e limitações funcionais. O tratamento conservador, geralmente, tem seus resultados avaliados com base na função e intensidade da dor referida pelo paciente ${ }^{(1)}$.

É importante caracterizar e quantificar a dor no ombro para que o indivíduo portador de tal afecção possa assumir posturas corretas dos membros superiores, durante atividades laborativas e cotidianas. As condutas adotadas pelos profissionais da saúde, baseadas na intensidade da dor, poderiam prevenir a evolução crônica da síndrome ${ }^{(2)}$.

Frente a isto, um estudo recente objetivou produzir uma versão brasileira de um questionário destinado a avaliar a dor, a satisfação e a função dos indivíduos que apresentam condições dolorosas musculoesqueléticas do ombro. Este instrumento, o Penn Shoulder Score (PSS), foi desenvolvido em 1999 para analisar indivíduos com disfunção de ombro. Ele consiste de uma escala de 0 a 100 pontos que inclui três domínios: dor, satisfação e função. A pontuação máxima do PSS indica ausência de dor, alta satisfação e boa função ${ }^{(4)}$.

A utilização de uma escala, que possibilite ao profissional da saúde e ao indivíduo acometido pela SOD realizar uma avaliação da funcionalidade, satisfação e da dor do braço afetado, é fundamental para acompanhar a evolução da condição do paciente submetido a um tratamento para tal disfunção.

Dentre as propostas de tratamento para a SOD, a acupuntura é hoje uma modalidade de intervenção sugerida pelas equipes médicas. Dentro desta modalidade, temos a acupressão como uma delas. Ela é definida como um método de tratamento terapêutico que consiste em uma antiga arte de cura da Medicina Tradicional Chinesa (MTC), que usa uma pressão para estimular pontos reflexos, cuja propriedade é de restabelecer o equilíbrio, alcançando assim resultados terapêuticos. Tais pontos estão estreitamente relacionados com os pontos utilizados pela acupuntura. A diferença entre ambos é que, na acupuntura, estes pontos são estimulados com a inserção de agulhas, enquanto que, na acupressão, utiliza-se a pressão firme, ou dos dedos das mãos, ou de outras estruturas, para pressionar a região desejada ${ }^{(5-9)}$.

A acupressão pode também ser utilizada na região auricular, uma vez que a orelha externa é um dos vários microssistemas e está repleta de pontos de acupuntura que correspondem a todos os órgãos e estruturas do corpo, ou seja, o corpo humano está representado pela orelha como um mapa formado por acupontos. Esta técnica é também denominada de auriculoterapia ${ }^{(5-6,8)}$.

A neuroanatomia e a neurofisiologia não são capazes de explicar completamente o mecanismo de ação, tanto da acupuntura como da auriculoterapia. As explicações são justificadas porque a aurícula possui uma grande quantidade de inervação derivada dos nervos trigêmeos, do facial, do vago, os auriculares maiores e os occipitais maiores e menores. Isso significa que em toda a região da orelha externa existem pontos associados com uma inervação ligada ao cérebro e este por sua vez unido pela rede do sistema nervoso, a determinado órgão ou região do corpo, comandando suas funções. A relação ponto auricular-cérebro-órgão é que tornam a auriculoterapia compatível como tratamento das mais variadas patologias ${ }^{(8)}$.

Alguns estudos desenvolvidos nessa área buscam avaliar esse método terapêutico em diferentes situações, seja para: demonstrar sua efetividade na analgesia de pacientes com hérnia de disco lombar ${ }^{(10)}$; na capacidade de aprendizagem e memória de ratos modelo com a doença de Alzheimer ${ }^{(11)}$; na redução da dor pélvica e lombar secundária à gravide $z^{(12)}$; como terapia adjuvante na artrite reumatóide ${ }^{(13)}$; no controle dos transtornos generalizados de ansiedade ${ }^{(14)}$; no tratamento de asma bronquica na infância(15); ou até mesmo no controle da náusea e vômitos em pacientes de câncer de mama submetidos a quimioterapia adjuvante ${ }^{(9)}$.

Nessas pesquisas, os autores discutem algumas questões sobre a auriculoterapia, incluindo a sua eficácia, a relação do seu mecanismo de ação sobre as doenças, a seleção exata de acupontos auriculares e a necessidade de mais pesquisas sobre a aplicação deste método. Em todos os estudos, os autores apontam que a acupuntura auricular tem as características de alta taxa de efetividade, taxa de cura e baixa taxa de recorrência da afecção. Outro ponto discutido, em tais estudos, é em relação ao tempo de tratamento, tanto com o uso de agulhas, como com o uso da acupressão auricular. $\mathrm{O}$ tempo estimado de tratamento nos estudos variou entre 10 a 12 semanas $^{(9-15)}$.

Reconhecendo a auriculoterapia como um importante instrumento de analgesia e cura, este estudo busca avaliar os resultados do uso da acupressão auricular, para uma portadora do Diagnóstico de Enfermagem de Dor Crônica, secundário a Síndrome do Ombro Doloroso.

\section{OBJETIVO}

Avaliar os resultados da acupressão auricular quando usada como terapia no tratamento da Dor crônica, secundária à Síndrome do Ombro Doloroso, quanto ao efeito analgésico e terapêutico satisfatório e o tempo estimado de tratamento para obtenção desses resultados.

\section{MÉTODO}

Para a obtenção do objetivo proposto foi realizado um Estudo de Caso, modalidade de pesquisa entendida como a escolha de um objeto de estudo definido pelo interesse em 
casos individuais. Ela busca a investigação de um caso específico, bem delimitado, contextualizado em tempo e lugar para que se possa realizar uma busca circunstanciada de informações ${ }^{(16-17)}$.

Conforme os objetivos dessa pesquisa, este Estudo de Caso é classificado como instrumental, pois objetiva examinar um caso para se compreender melhor a ação da auriculoterapia na SOD, servindo assim como base para orientar estudos ou ser instrumento para pesquisas posteriores ${ }^{(16)}$.

Este estudo teve como intervenção a acupressão auricular e como indicador de resultado o questionário Penn Shoulder Score (PSS - Brasil). Este instrumento foi desenvolvido para analisar indivíduos com disfunção de ombro, consistindo de uma escala de 100 pontos que inclui três domínios: Dor, Satisfação e Função.

Os domínios de dor e satisfação apresentam, respectivamente, três itens e um item avaliados por meio de uma Escala de Avaliação Numérica (EN) de 0 a 10, sendo que 0 corresponde à ausência de dor e a não satisfeito, enquanto 10 corresponde à pior dor possível e a muito satisfeito. $O$ domínio de função contém vinte itens, graduados em uma escala de Likert de quatro pontos, variando de 0 , que significa "não consigo fazer de forma alguma", a 3, "sem dificuldade", com pontuação máxima de 60 pontos. A pontuação do PSS varia de 0 a 100 pontos, com a pontuação máxima indicando ausência de dor, alta satisfação e boa função(4).

A avaliação dos itens Dor, Satisfação e Função do questionário PSS - Brasil foi realizada antes do primeiro atendimento, como critério de inclusão e levantamento do escore das consequências das disfunções de ombro e foi re-aplicado na oitava, na décima e na décima segunda semana de tratamento. As três perguntas do item Dor e a pergunta do item Satisfação do questionário foram aplicadas semanalmente.

A pesquisa foi realizada no local de trabalho da participante da pesquisa.

Os critérios para que a participante fosse incluída nesse estudo foram: ter acima de 18 anos; ter concordância por escrito para a participação na pesquisa; ter dor e limitação nos movimentos do ombro; ser avaliada com o questionário PSS - Brasil e ter a pontuação positiva para dor e disfunção de ombro; não estar fazendo uso de nenhuma outra terapêutica para o tratamento ou controle da dor (medicação analgésica, anti-inflamatória, corticosteróides, fisioterapia, massagens e acupuntura sistêmica); não estar em programação de férias durante o período de intervenção; não estar grávida.

Os dados foram analisados qualitativa e quantitativamente. Os dados quantitativos foram armazenados no programa Excel for Windows Explorer ${ }^{\circledast}$ e analisados estatisticamente com cálculos simples. Foram utilizadas análises descritivas (número e percentual), objetivando caracterizar e analisar a amostra.

A pesquisa teve aprovação da Instituição no qual a participante trabalha (ofício DS-009/06/11) e do Comitê de Ética da Universidade Cruzeiro do Sul (protocolo CE/UCS-075/2011). Foi explicado para a participante o objetivo do estudo e lhe apresentado o Termo de Consentimento Livre e Esclarecido, solicitando a leitura e oficialização da pesquisa. Foi reafirmado que a participação dela era voluntária, garantindo a permanência dos dados sob a guarda dos pesquisadores. Foi informado ainda que, se em algum momento, ela decidisse não continuar no estudo, poderia nos contatar, a fim de que excluí-la da pesquisa.

\section{APRESENTAÇÃO DO CASO}

MOS, casada, 42 anos é policial militar do Estado de São Paulo há 23 anos. Refere dupla jornada de trabalho: no quartel e em sua casa, onde realiza as atividades de organização e limpeza do lar. Durante o seu período na Polícia Militar, ela migrou de um setor para outro, desenvolvendo atividades de patrulhamento na maior parte da sua carreira militar. Relata que entre as atividades laborativas que mais utiliza os membros superiores, os treinamentos onde tem que ficar "em guarda" (posição ereta do corpo, com os membros superiores voltados para a parte posterior, na região dorsal), é a mais desgastante fisicamente, pois tem que permanecer durante horas na mesma posição. Após esse longo período "em guarda", o policial militar completa seu treinamento e apresentações com a "marcha", no qual faz movimentos com os membros superiores de repetição.

Atualmente, MOS trabalha em um setor administrativo da corporação, permanece longos períodos trabalhando no computador e cumpre, de tempos em tempos, escalas de plantão de patrulhamento e apresentações em solenidades.

Ela relata que, há aproximadamente um ano e dois meses, vinha sentindo um desconforto nos dois ombros, porém tal desconforto não Ihe causava limitação importante. Esse desconforto era descrito como uma dor de intensidade leve. Ao participar em abril de 2010 de uma solenidade da Polícia Militar, MOS permaneceu durante mais de seis horas em treinamento, tendo que ficar na mesma posição e com os braços para trás. Afirma que, a partir desse dia, ela começou a sentir fortes dores nos dois ombros, sendo que a dor era pior no ombro esquerdo. A dor foi tão intensa, que ela procurou auxílio médico, sendo afastada das suas atividades. Foi diagnosticada, após exame de tomografia computadorizada da coluna cervical, uma artrose cervical com irradiação para o ombro. Fez sessões de fisioterapia e uso de anti-inflamatórios não esteroides. Como não apresentou melhora após esse tratamento, fez uso de corticosteróides que aliviaram os sintomas.

Relata que a dor em seus ombros passa por períodos de melhora e piora. Diz que quando exerce atividades como limpar a casa ou ficar em forma, no dia subsequente, ela não consegue trabalhar e tem que fazer uso de algum analgésico ou relaxante muscular.

Desde o primeiro pico de piora, ela não consegue dormir à noite, diz que tem "sono picado", pois acorda com dor quando, inconscientemente, gira na cama, caindo em cima do ombro mais afetado. Afirma ainda que no dia posterior a sua noite de sono interrompida, ela percebe que fica mal humorada e irritada com qualquer situação.

O Diagnóstico de Enfermagem para MOS é o de Dor crônica. A sua pontuação segundo o questionário PSS - Brasil teve o seguinte escore conforme o Quadro 1: 
Quadro 1 - Primeira avaliação de MOS, São Paulo, 2011.

\begin{tabular}{|c|c|c|c|c|}
\hline \multicolumn{4}{|c|}{ Pontuação de MOS segundo o questionário PSS - Brasil } \\
\hline Data & $\begin{array}{c}\text { PSS } \\
\text { Dor }\end{array}$ & $\begin{array}{c}\text { PSS } \\
\text { Satisfação }\end{array}$ & $\begin{array}{c}\text { PSS } \\
\text { Função }\end{array}$ & Pontuação total \\
\hline $25 / 03 / 2011$ & $\mathbf{1 3 / 3 0}$ & $\mathbf{1 / 1 0}$ & $\mathbf{3 0 / 6 0}$ & $\mathbf{4 4 / 1 0 0}$ \\
\hline
\end{tabular}

Após a identificação por meio do instrumento Penn Shoulder Score - Brasil que MOS apresenta alteração na Dor, Satisfação e Função em ambos os ombros, foi proposto o tratamento descrito abaixo.

\section{PROCEDIMENTO}

A intervenção consistiu no método de acupressão auricular com esferas de cristais, por serem consideradas neutras, polidas, com 1,5 mm de diâmetro, presas em esparadrapo, produzindo uma ação mecânica sobre o acuponto desejado. As esferas foram colocadas sobre os pontos descritos abaixo com auxílio de uma pinça mosquito, após limpeza de toda a região auricular com álcool a $70 \mathrm{P} / \mathrm{V}$. Os pontos escolhidos foram direcionados segundo os descritos pela Medicina Tradicional Chinesa (MTC) para o tratamento da SOD conforme segue:

A. Triângulo Cibernético ou Auriculocibernético: é indicado para iniciar qualquer tipo de programa de terapia auricular $^{(5,8)}$. É constituído pela ordem dos seguintes pontos:

$\checkmark$ Shenmen, têm como função predispor o tronco e o córtex cerebral a receber e decodificar os reflexos dos pontos que serão utilizados, a provocar no cérebro a produção de cargas de hormônios naturais do tipo "endorfinas", produzindo efeito sedativo ${ }^{(5,8)}$;

$\checkmark$ Rim: regula os ossos e estimula: a filtragem do sangue pelos rins, eliminando mais metabólitos; as funções do sistema respiratório a aumentar o processo de metabolismo do oxigênio; o aumento da secreção de hormônios pelas glândulas endócrinas e as funções dos órgãos excretores, inclusive das glândulas sebáceas e sudoríparas $^{(5,8)}$;

$\checkmark$ Simpático: tem como mecanismo de ação acelerar e regular as atividades do sistema neurovegetativo, equilibrando as funções do simpático e do parassimpático, nesse sentido ao equilibrar as funções do sistema nervoso autônomo provoca equilíbrio geral no organismo. Estimula as funções da medula óssea, bem como o metabolismo de cálcio, agindo sobre o tecido ósseo e o periósteo equilibrando sua formação ou regeneração. Provoca vasodilatação e age sobre os tecidos musculares provocando ação anti-inflamatória, relaxamento ou tonificação das fibras do sistema músculo-tendinoso ${ }^{(5,8)}$.

B. Ombro: têm como indicações terapêuticas as Bursites, Tendinites, Luxação do ombro, Dores subaxilares, Paralisia dos membros superiores, Sequelas de Esclerose lateral amiotrófica $^{(5,8)}$; $\checkmark$ Articulação do ombro: têm como indicações terapêuticas as Bursites, Calcificações, Tendinites, Reumatismos, Artrites, Fraturas, Analgesia e Anestesia da região ${ }^{(5,8)}$;

$\checkmark$ Clavícula: têm como indicações terapêuticas as dores na região, fratura de clavícula, inflamações dos músculos da área da clavícula, ruptura de tendões e luxações ${ }^{(5,8)}$;

O ciclo de tratamento consistiu em doze semanas.

A paciente foi orientada a estimular as esferas sobre os acupontos em média cinco vezes ao dia (ao levantar-se, próximo da hora do almoço, no meio da tarde, próximo da hora do jantar e antes de dormir), com duração de um minuto em cada ponto. As esferas permaneceram na orelha durante uma semana. A cada sessão semanal foi rodiziado a orelha, alternado entre orelha direita e esquerda para evitar a formação de lesões até o final do ciclo de tratamento. Todos os atendimentos foram realizados pela pesquisadora para que se mantivesse um parâmetro fidedigno dos acupontos selecionados.

\section{RESULTADOS}

Após a primeira aplicação das esferas de cristais MOS relatou que os acupontos estavam "queimando", ela sentia a sua orelha "pegando fogo". Tal sensação se manteve nas sessões subsequentes.

Após uma semana de tratamento, a paciente relatou que melhorou muito o seu sono, chegando dormir quatro horas sem interrupção. De acordo com o Quadro 2 pode-se observar que houve significativa melhora da pontuação da Dor e Satisfação de MOS. O escore de Dor passou de 13 para 25, uma melhora de $40 \%$. Já o item Satisfação a melhora foi de $70 \%$ em apenas uma semana de tratamento.

Na terceira semana MOS apresentou uma pequena lesão no ponto do Shenmen e no ponto da Clavícula. Frente a isto, foi aplicada a solução de Cavilon ${ }^{\circledast}$ em todo o pavilhão da orelha externa. Ela trouxe como principal resultado nessa semana a acentuada melhora do seu padrão de sono. Referiu que conseguiu manter durante toda a semana um padrão periódico de descanso durante a noite, despertando ao amanhecer com a sensação de satisfação e sono restaurador. De acordo com o Quadro 2 pode-se observar que a melhora da pontuação da Dor e Satisfação de MOS em relação a segunda semana ainda foi de forma crescente, revelando em relação a Dor, uma melhora entre a primeira e a terceira semana de tratamento de $50 \%$. Já em relação a Satisfação, a melhora de MOS foi de $80 \%$.

No seu quarto atendimento foi observado que as lesões apresentadas no dia 08/04/2011 estavam cicatrizadas. MOS relatou que durante a semana ela evoluiu com Dor tipo "queimação" no ombro esquerdo, o mais afetado. Quando questionada a descrever as intercorrências que ocorreram durante essa semana, ela relatou que sua filha acidentou-se na escola e que tal situação desencadeou um episódio de extremo estresse, ficou com raiva da professora de sua filha, pois acreditou que ela foi diretamente responsável pelo acidente. Essa intercorrência influenciou na evolução do seu tratamento. Isso é demonstrado pelos escores das pontuações de Dor e Satisfação, ambos declinaram 20\% em relação a semana anterior (Quadro 2). 
Quadro 2 - Avaliações de MOS segundo o questionário PSS - Brasil, São Paulo, 2011.

\begin{tabular}{|c|c|c|c|c|c|c|c|c|}
\hline Avaliação & Data & Dor & $\begin{array}{l}\text { Percentual } \\
\text { de melhora }\end{array}$ & Satisfação & $\begin{array}{l}\text { Percentual } \\
\text { de melhora }\end{array}$ & Função & Pontuação total & $\begin{array}{l}\text { Percentual } \\
\text { de melhora }\end{array}$ \\
\hline $1^{\mathrm{a}}$ & $25 / 03 / 2011$ & $13 / 30$ & & $1 / 10$ & & $30 / 60$ & $44 / 100$ & \\
\hline $2^{\mathrm{a}}$ & 01/04/2011 & $25 / 30$ & $40,00 \%$ & $8 / 10$ & $70,00 \%$ & Não aplicado & Não aplicado & \\
\hline $3^{\mathrm{a}}$ & 08/04/2011 & $28 / 30$ & $50,00 \%$ & $9 / 10$ & $80,00 \%$ & Não aplicado & Não aplicado & \\
\hline $4^{\mathrm{a}}$ & $15 / 04 / 2011$ & $22 / 30$ & $30,00 \%$ & $7 / 10$ & $60,00 \%$ & Não aplicado & Não aplicado & \\
\hline $5^{\mathrm{a}}$ & $29 / 04 / 2011$ & $27 / 30$ & $46,67 \%$ & 9/10 & $80,00 \%$ & Não aplicado & Não aplicado & \\
\hline $6^{\mathrm{a}}$ & 06/05/2011 & $27 / 30$ & $46,7 \%$ & $9 / 10$ & $80,00 \%$ & Não aplicado & Não aplicado & \\
\hline $7^{\mathrm{a}}$ & $13 / 05 / 2011$ & $23 / 30$ & $33,3 \%$ & $9 / 10$ & $80,00 \%$ & Não aplicado & Não aplicado & \\
\hline $8^{\mathrm{a}}$ & 20/05/2011 & $27 / 30$ & $46,7 \%$ & $9 / 10$ & $80,00 \%$ & $37,77 / 60$ & $73,77 / 100$ & $29,7 \%$ \\
\hline $9^{a}$ & $27 / 05 / 2011$ & $26 / 30$ & $43,3 \%$ & $9 / 10$ & $80,00 \%$ & Não Aplicado & Não aplicado & \\
\hline $10^{\mathrm{a}}$ & 03/06/2011 & $28 / 30$ & $50,00 \%$ & $9 / 10$ & $80,00 \%$ & $38,77 / 60$ & $75,77 / 100$ & $31,7 \%$ \\
\hline $11^{\mathrm{a}}$ & 10/06/2011 & $27 / 30$ & $46,7 \%$ & $9 / 10$ & $80,00 \%$ & Não Aplicado & Não aplicado & \\
\hline $12^{\mathrm{a}}$ & $17 / 06 / 2011$ & $27 / 30$ & $46,7 \%$ & $9 / 10$ & $80,00 \%$ & $42,35 / 60$ & $78,35 / 100$ & $34,3 \%$ \\
\hline
\end{tabular}

No quinto e sexto atendimento, os escores de Dor e Satisfação apresentaram uma melhora de $46,7 \%$ e $80 \%$ respectivamente. Nesse mesmo período, MOS participou ativamente da mudança de seu departamento, exercendo muita atividade física com os membros superiores. Em decorrência disso, relatou dores em ambos os ombros, porém associa essas dores com estafa muscular e não relaciona com a mesma dor que sentia nos ombros antes de iniciar a intervenção dessa pesquisa.

Na sétima semana houve uma redução do escore de Dor de $46,7 \%$ para $33,3 \%$, no entanto, o escore de Satisfação manteve-se inalterado. Ao ser questionada sobre os acontecimentos da sua semana, MOS não correlacionou nenhum fato novo que justificasse a redução do seu escore da Dor.

Na oitava semana foi reaplicado o questionário PSS - Brasil incluindo o item função. Utilizando a primeira avaliação como parâmetros iniciais de comparação, pode-se verificar uma melhora na pontuação total do questionário PSS - Brasil de MOS em 29,7\%. Já em relação as pontuações isoladas dos itens Dor e Satisfação, os escores voltaram a se equiparar aos mesmos das semanas de 29/04/2011 e 06/05/2011 conforme demonstra o Quadro 2.

$\mathrm{Na}$ nona semana de atendimento foi observado que nos acupontos do ombro, articulação do ombro e clavícula da orelha direita houve um aumento na pigmentação desses locais para um tom castanho, porém sem o aparecimento de solução de continuidade. A pontuação do item Dor reduziu 1 ponto e o item Satisfação se manteve inalterado, conforme revela o Quadro 2.

Na décima semana foi reaplicado o questionário PSS Brasil para os itens Dor, Satisfação e Função. Utilizando a primeira avaliação como parâmetros iniciais de comparação, pode-se verificar que houve uma melhora de MOS na pontuação total do questionário PSS - Brasil em 31,8\%. Porém, se compararmos os escores entre a oitava e a décima semana, a diferença foi de apenas $2 \%$.

Na décima primeira semana de atendimento não houve alteração significativa do escore de Dor e a pontuação do item Satisfação se manteve inalterado nas últimas sete semanas. O escore do item Dor sofreu alterações mínimas sem relevância estatística.

O décimo segundo e último atendimento foi realizado em 17/06/201 e foi reaplicado o questionário PSS - Brasil completo conforme revela o Quadro 2. Comparando a pontuação total do questionário PSS - Brasil de MOS entre a primeira semana e a décima segunda semana de atendimento, pode-se observar uma melhora de $34,3 \%$. Se compararmos a oitava semana com a décima segunda houve uma diferença de 4,7\%.

\section{DISCUSSÃO}

A disfunção do ombro de MOS, segundo o instrumento PSS - Brasil, antes do início do tratamento, teve uma pontuação baixa - 44/100, indicativo de alterações significativas quanto a Dor, Satisfação e Função dos seus ombros. Ao contrário de outros questionários ou escalas para avaliação da Dor, o PSS - Brasil possui uma pontuação que varia de 0 a 100 pontos, com a pontuação máxima indicando ausência de Dor, alta satisfação e boa função.

Durante todo o tratamento, MOS sempre relatava que, após a colocação das esferas, os pontos selecionados ficavam "queimando", sentia que a sua orelha estava "pegando fogo". Essa sensação é explicada pela hiperestimulação da rede nervosa e ativação da circulação na rede vascular da orelha que aciona e excita o fluxo energético ${ }^{(18)}$. Os autores dessa temática descrevem esses sintomas como sendo reações normais e esperadas, aparecem em aproximadamente $80 \%$ dos pacientes 
e estão ligadas ao êxito do tratamento, e caso o paciente não refira ou não perceba essas respostas, o terapeuta deverá rever se os acupontos utilizados são os mais indicados ${ }^{(18)}$.

Uma das principais queixas de MOS, em relação a sua disfunção, era a insônia. Este problema é descrito segundo a taxonomia II de NANDA, 2010, como "distúrbio na quantidade e na qualidade do sono que prejudica o funcionamento normal de uma pessoa"(19). Este diagnóstico pode ser evidenciado pela paciente, pois ela apresentava características bem definidas como sensação de sono não restaurador, alterações do humor e dificuldade para permanecer dormindo. Esses sintomas estão estreitamente relacionados com a Dor que MOS sentia ao mover-se na cama durante a noite e, inconscientemente, ficar em cima do ombro mais afetado.

Estudos descrevem o aparecimento dos distúrbios do sono e da influência que o binômio sono-vigília nas doenças em que a Dor é presente. Falam também da importância de, além do tratamento álgico, focar em intervenções específicas para a promoção do sono restaurador ${ }^{(20)}$. Tais preocupações se dão pelo fato de comprovações, por intermédio de estudos clínicos e experimentais, tanto em humanos como em animais, que há uma relação recíproca entre sono e Dor, baseados em explicações neurofisiológicas de que áreas do cérebro envolvidas com a percepção da Dor também estão envolvidas com a geração e manutenção do sono ${ }^{(20)}$. Nesse sentido, essa relação transforma-se em um ciclo vicioso autossustentado, ou seja, a presença da Dor, principalmente a crônica, gera constantes estímulos sensoriais que ativam e mantém áreas do sistema nervoso central, responsáveis pelo estado de alerta, enquanto rejeita áreas responsáveis pelo início e manutenção do sono ${ }^{(20)}$. O resultado desse processo é o aumento dos distúrbios do sono à medida que a Dor também aumenta. As consequências são devastadoras, pois a ausência de funções restaurativas e reparativas do sono levam a um retardo no processo de cura, aumentando ainda mais a Dor ${ }^{(20)}$.

Pode-se observar que esse ciclo vicioso foi apresentado pela paciente deste estudo e interrompido na terceira semana de tratamento a partir do momento em que a Dor foi controlada por intermédio da auriculoterapia. Em apenas três sessões a pontuação da Dor de MOS melhorou em 50\% o que causou o desaparecimento do diagnóstico de insônia.

A partir do momento em que MOS diminui a sua Dor e melhorou a qualidade do seu padrão de sono, consequentemente também melhorou seu humor, desempenho no trabalho e atividades rotineiras. Nesse mesmo período, a sua Satisfação em relação ao ombro também apresentou índices elevados até o final do tratamento.

Uma reação adversa apresentada por MOS foi o aparecimento de pequenas lesões nos pontos do Shenmen e Clavícula na terceira semana de tratamento. Tais lesões podem ter ocorrido em decorrência do excesso de pressão exercida por MOS durante os estímulos diários, e está relacionada com os riscos da auriculoterapia ${ }^{(18)}$. O repouso da orelha feito durantes as sessões e seus ciclos objetivam reduzir ou evitar essas intercorrências.

Surgiram nos pontos do ombro, articulação do ombro e clavícula de MOS aumento da pigmentação, transformando-se em uma cor marrom, que segundo alguns autores, está relacionado por estagnação energética nas doenças crônicas em andamento ou ainda naquelas que já foram "curadas" $(5,18)$. Nesse sentido, pode-se correlacionar essas afirmativas com as alterações apresentadas por MOS. Elas começaram a ser notadas na nona semana de tratamento, no período em que seus escores do PSS - Brasil, comparado com o início do tratamento, teve uma melhora na pontuação total em 29,8\%.

É importante comparar a intercorrência vivenciada por MOS durante o período em que esteve participando da pesquisa, na quarta semana, com a evolução do seu tratamento. Segundo a MTC, o homem é visto como um organismo único, sem divisões. As emoções e as estruturas físicas fazem parte de um só corpo. Nesse sentido, cada tecido, emoção ou órgão interno, tem um papel fundamental e específico na manutenção da saúde do ser humano ${ }^{(7,18)}$. Sendo assim, "qualquer situação que venha a prejudicar o normal funcionamento dessas estruturas acarreta o surgimento de sinais e sintomas, indicativos da presença de desequilíbrio, as chamadas síndromes energéticas" ${ }^{\prime 18)}$.

MOS vivenciou a raiva nesse período quando soube do acidente da sua filha na escola. Segundo a MTC a fúria afeta o elemento Madeira, desequilibrando-o. Tal elemento é responsável pelo "movimento" e controla as articulações do $\operatorname{corpo}^{(7,18)}$. A consequência disso foi o declínio na evolução do seu tratamento, sendo demonstrado pela redução das pontuações de Dor e Satisfação.

A SOD de MOS foi desencadeada pela artrose, que teve início na coluna cervical e, posteriormente, evoluiu para as articulações de ambos os ombros. No ocidente, a artrose é definida como uma doença de toda a articulação (cartilagem, ligamentos, sinóvia e osso), a lesão inicial costuma ser na cartilagem articular. Ela tem um forte componente genético e, na maioria das vezes, tem a sobrecarga mecânica como um iniciador do processo de lesão da cartilagem, que acaba evoluindo para um ciclo vicioso inflamatório, perpetuando a degradação articular (21).

Fazendo uma correlação com a MTC, a artrose causada por estresse físico consome a energia do Rim, responsável por controlar os líquidos orgânicos e determinar as condições dos ossos, sendo assim, compromete a quantidade do líquido sinovial e as restaurações ósseas das articulações ${ }^{(5-7)}$.

Nesse sentido, o tratamento proposto neste estudo para MOS foi o de estimular pontos reflexos na orelha com um material neutro (esferas de cristais), para que o organismo reagisse equilibrando os mecanismos compensatórios responsáveis pela mobilidade da articulação do ombro, bem como o de induzir o processo restaurador da articulação, conforme descrito no item procedimento.

Até algumas décadas atrás, o tratamento da osteoartrite ou artrose ficou limitado ao uso de analgésicos simples, anti-inflamatórios, medidas físicas, infiltrações com corticóides e, nos casos refratários e mais graves, o tratamento cirúrgico. Com o avanço de pesquisas sobre essa temática e o esclarecimento das vias inflamatórias envolvidas induziram os médicos a adotarem outros medicamentos para o tratamento e controle de tal afecção ${ }^{(21)}$. Vale ressaltar que os estudos ainda 
descartam a possibilidade de cura da artrose, no entanto, esses mesmos estudos trazem para a discussão a existência de drogas modificadoras da doença, que têm a capacidade de alterar a evolução na degeneração articular, retardando sua progressão, podendo até torná-la assintomática e, desse modo, evitando uma parcela dos procedimentos cirúrgicos de salvação ${ }^{(21)}$. Porém, tais pesquisas ainda estão em andamento, sem comprovação de resultados favoráveis em longo prazo, uma vez que a artrose é uma doença crônica e de evolução lenta ${ }^{(21)}$. Atualmente ainda nos deparamos com prescrições de medicações de ação antiinflamatórias que apresentam efeitos colaterais devastadores, como por exemplo, o Diclofenaco de Sódio, que lista inúmeros efeitos colaterais.

A medicação citada por MOS, de que ela fazia uso periódico antes do início da pesquisa, tem como uma de suas composições o diclofenaco sódico. A partir da segunda semana de tratamento, com a melhora do item Dor do PSS-Brasil, MOS aboliu o uso de drogas anti-inflamatórias para o controle da Dor.

Ao comparar o tempo de tratamento da auriculoterapia na SOD, percebe-se que a diferença entre a melhora de MOS da oitava para a décima semana de tratamento é de apenas $2 \%$, não representando alteração estatística significativa. Porém, quando se aumenta mais quatro semanas, ou seja, mais quatro sessões, esse diferença aumenta para 4,6\%.

Comparando somente os itens de Dor e Satisfação, pode-se observar que, após a oitava semana, o item Satisfação manteve-se inalterado; já o item Dor teve pequenas oscilações, sem relevância estatística. $\mathrm{O}$ item Função teve uma melhora de 7,77 pontos entre a primeira avaliação e a oitava, essa diferença aumentou em apenas 1 ponto entre a oitava e a décima semana. Ao compararmos a primeira avaliação com a décima segunda a melhora atingiu uma diferença de 12,35 pontos. Ou seja, mesmo MOS não fazendo uso de medicações classificadas como "drogas específicas" para o tratamento da osteoartrite, a função de seus ombros teve uma evolução significativa.

\section{CONCLUSÃO}

Reconhecendo cada vez mais a auriculoterapia como uma importante técnica terapêutica, este estudo contribuiu com dados pontuais e relevantes para o entendimento de que essa modalidade da MTC pode e deve ser utilizada pelos enfermeiros como tratamento das alterações osteoarticulares dos ombros com baixo índice de efeitos colaterais ou adversos. Isso se deve aos resultados apresentados nessa pesquisa, em que se evidenciou uma melhora da paciente em questão, em $34,3 \%$ da sua disfunção classificada como SOD. Pode-se concluir também que o tratamento com a auriculoterapia pode abolir o uso de drogas analgésicas e anti-inflamatórias e seus efeitos deletérios.

Pode-se afirmar que a seleção dos pontos utilizados na pesquisa pode ser classificada como um protocolo de tratamento para a SOD, e o tempo de tratamento indicado nesse estudo é de doze sessões. As sessões deverão ocorrer semanalmente, alternando entre as duas orelhas para evitar lesões e fadiga auricular em decorrência do processo de acomodação do sistema nervoso.

O instrumento (PSS - Brasil) que foi adotado como indicador de resultado, permitiu a compreensão e análise dos resultados de forma objetiva segura.

É importante ressaltar que a Dor gera inúmeros transtornos para a pessoa que a vivencia, como por exemplo, a insônia. Portanto, quando se assume o papel de terapeuta, deve-se abrir o campo de observação para perceber a totalidade do indivíduo sob os cuidados e conseguir fazer correlações entre os sintomas e as disfunções orgânicas. Isso significa que nem sempre é necessário estimular o organismo com inúmeros comandos, como na auriculoterapia, inserir várias esferas ou agulhas para tentar tratar todos os sintomas. Devemos lembrar dos preceitos da MTC que ensina que, ao equilibrar um elemento, os demais vão também se equilibrando em um processo harmônico e gradual.

Espera-se ter contribuído para demonstrar a efetividade da auriculoterapia como uma ferramenta de intervenção rápida e eficaz no tratamento das afecções osteoarticulares para os terapeutas e enfermeiros habilitados a exercer tal atividade.

No entanto, é importante ressaltar que isto é o início da compreensão desta possibilidade terapêutica. Abre-se um leque de possibilidades de investigação para a validação destas propostas, bem como a apresentação de outros acupontos e técnicas de estímulos.

Deve-se pensar que é importante reconhecer os resultados alcançados, porque eles podem ser a base para o desenvolvimento exploratório de outras pesquisas sobre essa temática. Assim, este trabalho pode servir como um convite para que os profissionais que atuam nas Terapias Alternativas e Complementares ampliem tais conhecimentos.

\section{REFERÊNCIAS}

1. Turtelli CM. Avaliação do ombro doloroso pela radiologia convencional. Radiol Bras 2001;34(4).

2. Garzedin DDS, Matos MAA, Daltro CH, Barros RM, Guimarães A. Intensidade da dor em pacientes com síndrome do ombro doloroso. Acta Ortop Bras 2008;16(3)165-167.

3. Ejnismann B, Monteiro GC, Uyeda LF. Ombro doloroso. Einstein (São Paulo) 2008;6(Suppl. 1):S133-S7.

4. Napoles BV, Hoffman CB, Martins J, Oliveira AS. Tradução e adaptação cultural do Penn Shoulder Score para a Língua Portuguesa: PSS-Brasil. Rev Bras Reumatol 2010;50(4):389-407.

5. Reichmann BT. Auriculoterapia: fundamentos de acupuntura auricular. 4. ed. Curitiba: Tecnodata; 2008.

6. Gerber R. Um guia prático de medicina vibracional. 10. ed. São Paulo: Cultrix; 2007.

7. Zhufan X. Prática da medicina tradicional chinesa. São Paulo: Ícone; 2009. 
8. Souza MP. Tratado de auriculoterapia. Brasília: FIB; 2007.

9. Chung WT. Estudo prospectivo do valor da acupuntura no controle da náusea e vômitos em pacientes de câncer de mama submetidos a quimioterapia adjuvante. São Paulo. Tese [Doutorado em Ciências] - Faculdade de Medicina da USP; 2007.

10. Liu EJ, Jia CS, Li XF, Ma XS, Shi J. Study on the effects of the auricular acupuncture with different manipulations for rapid analgesia on the patients with lumbar disc hernia. Zhongguo Zhen Jiu 2010;30(1):35-9.

11. Miao $\mathrm{T}$, Jiang $\mathrm{TS}$, Dong $\mathrm{YH}$, Jiang NC. Effects of auricular acupuncture on the memory and the expression of ChAT and GFAP in model rats with Alzheimer's disease. Zhongguo Zhen Jiu 2009;29: (10):827-32.

12. Wang $S M$, Dezinno $P$, Lin EC, Lin $H$, Yue J, Berman MR, et al. Auricular acupuncture as a treatment for pregnant women who have low back and posterior pelvic pain: a pilot study. Am J Obstet Gynecol 2009;201(3):271.e1-9.

13. Bernateck M, Becker M, Schwake C, Hoy L, Passie T, Parlesak A, et al. Adjuvant auricular electroacupuncture and autogenic training in rheumatoid arthritis: a randomized controlled trial. Auricular acupuncture and autogenic training in rheumatoid arthritis. Forsch Komplementmed
2008;15:(4):187-93.

14. Zaragoza Peña H, Faure Vidal AF. Auriculoterapia y fitoterapia em los transtonos generalizados de ansiedad. Rev Hosp Psiquiátr la Habana 2008;5(1).

15. Hervis Lee T, Valdés Pacheco E, Tallec Afonso A. La auriculopuntura con semillas en el tratamiento del asma bronquial en edad pediátrica. Rev Cuba Med Gen Integr 1999;15(4):419-425.

16. Severino AJ. Metodologia do trabalho científico. 23. ed. São Paulo: Cortez; 2007.

17. Gil AC. Métodos e técnicas de pesquisa social. São Paulo: Atlas; 1999.

18. Neves ML. Manual prático de auriculoterapia. Porto Alegre: Ed Do autor; 2009.

19. North American Nursing Diagnosis Association. Diagnósticos de enfermagem da NANDA: definições e classificação 2009-2011. Porto Alegre: Artmed; 2010.

20. Call-Schmidt TA, Richardson SJ. Prevalence of sleep disturbance and its relationship to pain in adults with chronic pain. Pain Manag Nurs 2003;4(3):124-33.

21. Rezende MU, Gobbi RG. Tratamento medicamentoso da osteoartrose do joelho. Rev Bras Ortop 2009;44(1). 


\section{ERRATA}

No volume 66, n 5, pp. 694-701, artigo Avaliação da acupressão auricular na Síndrome do Ombro Doloroso: estudo de caso, onde se lia como autor do artigo Ana Paula Zanelatto e sua respectiva afiliação e e-mail, leia-se:

\section{Ana Paula Zanelatto'}

' Universidade Cruzeiro do Sul, Curso de Graduação de Enfermagem. São Paulo-SP, Brasil. 